\title{
NOTES
}

\section{Solubility of Cellulose Acetate Prepared by Different Methods and Its Correlationships with Average Acetyl Group Distribution on Glucopyranose Units}

\author{
Kenji Kamide, Kunihiko OKAJIMA, Keisuke KowSAKA, \\ and Toshihiko MATSUI \\ Fundamental Research Laboratory of Fiber and \\ Fiber-Forming Polymers, Asahi Chemical Industry Co., Ltd., \\ 11-7 Hacchonawate, Takatsuki, Osaka 569, Japan
}

(Received May 1, 1987)

\begin{abstract}
KEY WORDS ${ }^{13} \mathrm{C} \mathrm{NMR} /$ Cellulose / Cellulose Acetate / Solubility / WaterSolubility / Degree of Substitution / Distribution of Degree of Substitution / $N, N$-Dimethylacetamide /
\end{abstract}

Very recently, experimental determination of the substituent group distribution towards three hydroxyl groups directly attached to $\mathrm{C}_{2}$, $\mathrm{C}_{3}$, and $\mathrm{C}_{6}$ positions in a glucopyranose unit of cellulose derivatives $\left\langle\mathrm{f}_{k}\right\rangle(k=2,3,6)$ has attracted keen attention, because this distribution has been shown to govern some important physical and physiological properties Kamide, Okajima, and their co-workers ${ }^{1-3}$ demonstrated that the anticoagulant activity of sodium cellulose sulfate ( $\mathrm{NaCS}$ ) against human blood and the absorbency of sodium carboxymethyl cellulose ( $\mathrm{NaCMC}$ ) against water and physiological saline water are significantly influenced by their $\left\langle\left\langle\mathrm{f}_{k}\right\rangle\right.$, in particular $\left\langle\mathrm{f}_{2}\right\rangle+\left\langle\mathrm{f}_{3}\right\rangle$ for $\mathrm{NaCS}$ and $\left\langle\mathrm{f}_{6}\right\rangle$ for $\mathrm{NaCMC}$. They ${ }^{1-3}$ also showed that $\mathrm{NaCS}$ and $\mathrm{NaCMC}$ with different $\left\langle\mathrm{f}_{k}\right\rangle$ can be prepared under adequate conditions, and cellulose acetate may not be an exception.

In 1981 Kamide and Okajima ${ }^{4}$ attempted to evaluate $\left\langle\mathrm{f}_{k}\right\rangle$ of cellulose acetate $(\mathrm{CA})$ by assigning three peaks in carbonyl carbon region to $\mathrm{C}_{2}, \mathrm{C}_{3}$, and $\mathrm{C}_{6}$ positions. Later, Miyamoto et al. ${ }^{5}$ estimated $\left\langle\mathrm{f}_{k}\right\rangle$ of CA based on different assignments of the peaks of three carbonyl carbons. Kowsaka and his collaborators ${ }^{6}$ showed that the theoretical basis of the assignment of carbonyl carbon peaks in the past studies on CA, especially when the total degree of substitution $\langle\mathrm{F}\rangle$ is below 3 , is not always very reliable, due to unavoidable overlapping of carbonyl carbon peaks originated from mono-, di-, and tri-substituted glucopyranose units. They succeeded to give a reasonable assignment of carbonyl carbon peaks of tri-substituted glucopyranose unit by applying a low-power selective spin decoupling method to acetyl methyl proton located at the specific carbon position. $^{6}$

Generally, CA having $\langle\mathrm{F}\rangle=1.7-2.5$ dissolves at room temperature readily in various organic solvents, including acetone, whereas it never dissolves in water at any temperature. Kamide et al. ${ }^{7}$ synthesized a series of CA with low $\langle\mathrm{F}\rangle$ by adopting a method of hydrolysis reaction of $\mathrm{CA}$ with $\langle\mathrm{F}\rangle=2.46$ with hydrochloric acid, confirming that CA prepared thus is water-soluble at "room temperature" in the $\langle\mathrm{F}\rangle$ range of $c a .0 .4$ to 0.9 . They established, for well-fractionated CA with $\langle F\rangle=0.49$, relationships between the limiting 
viscosity number $[\eta]$, the radius of gyration $\left\langle S^{2}\right\rangle_{z}{ }^{1 / 2}$ and the weight-average molecular weight $\bar{M}_{n}$, estimating the Flory's viscosity parameter $\Phi$, the unperturbed chain dimension A and the conformation parameter $\sigma$.

Four years later Miyamoto et $a .^{8}{ }^{\text {investi- }}$ gated the water-solubility of CA with low $\langle\mathrm{F}\rangle$, which are prepared by (1) the hydrolysis of CA sample with $\langle F\rangle=2.94$ in aq. acetic acid (D series) or in dimethylsulfoxide (DMSO) ( $\mathrm{H}$ series) and (2) the acetylation of cellulose dissolved in dimethylacetamide (DMAC)/lithium chloride $(\mathrm{LiCl})$ mixture $(\mathrm{A}$ series). They concluded that only CA samples with $\langle\mathrm{F}\rangle$ of $0.5-1.1$ prepared by method (1) are completely water-soluble at $20^{\circ} \mathrm{C}$. Based on analysis of $\left\langle\mathrm{f}_{k}\right\rangle$ of the water-soluble and -insoluble parts, estimated from ${ }^{13} \mathrm{C}$ and ${ }^{1} \mathrm{H}$ NMR spectra, they showed $\left.\left\langle\mathrm{f}_{6}\right\rangle\right\rangle\left\langle\left\langle\mathrm{f}_{3}\right\rangle\right.$ or $\left\langle\left\langle\mathrm{f}_{2}\right\rangle\right.$ for $\mathrm{H}$ and $\mathrm{A}$ series, but $\left\langle\mathrm{f}_{2}\right\rangle \simeq\left\langle\left\langle\mathrm{f}_{3}\right\rangle \simeq\right.$ $\left\langle f_{6}\right\rangle$ for $D$ series.

In this article we clarify the effects of the preparing conditions on $\left.\left\langle\mathrm{f}_{k}\right\rangle\right\rangle$ and the relative ratio of various substituted glucopyranose units of CA and to obtain the relations between $\langle\mathrm{F}\rangle,\left\langle\mathrm{f}_{k}\right\rangle(k=2,3$, and 6$)$ and the solubility of CA, discussing the dissolution mechanism of cellulose derivatives in solvents. For this purpose, we prepared nine CA samples by (1) a direct acetylation method of cellulose solution (one-step method) and (2) the acidhydrolysis of cellulose triacetate with $\langle\mathrm{F}\rangle=$ 2.92 (two-step method), as described in the previous paper, ${ }^{7}$ and measured ${ }^{13} \mathrm{C}$ NMR spectra of the samples in deuterated dimethylsulfoxide (DMSO- $d_{6}$ ).

\section{EXPERIMENTAL}

\section{Preparation of Cellulose Acetate Samples}

One-Step Method. Softwood pulp (cellulose I, the viscosity-average molecular weight $\bar{M}_{v}=$ $1.7 \times 10^{5}$, estimated from by the MarkHouwink-Sakurada (MHS) equation proposed by Brown and Wikström ${ }^{9}$ for cellulose in cadoxen solution at $25^{\circ} \mathrm{C}$ ) was immersed in $5 \mathrm{~N}$-sulfuric acid at $60^{\circ} \mathrm{C}$ for $80 \mathrm{~min}$ to give a hydrolyzed cellulose (cellulose I, $\bar{M}_{i}=8 \times 10^{4}$ ). Five $\mathrm{g}$ of this cellulose sample were dipped in $200 \mathrm{~g}$ of $N, N$-dimethylacetamide (DMAC) (guaranteed grade), heated at $165^{\circ} \mathrm{C}$ for $30 \mathrm{~min}$ with reflux, added $16 \mathrm{~g}$ of $\mathrm{LiCl}$ at $100^{\circ} \mathrm{C}$, cooled down to $20^{\circ} \mathrm{C}$ and maintained at that temperature with vital mechanical agitation to give optically a clear and colorless transparent solution. By adding various amounts of acetic anhydride and pyridine (as a catalyst) to the solution and stirring it at $20^{\circ} \mathrm{C}$ for $4-6 \mathrm{~h}$, CA samples were prepared. Table I summarizes the detailed preparing conditions, such as the amounts of acetic anhydride and pyridine added to the system and the reaction time. The resultant CA was completely precipitated with methanol, washed with methanol, dried in vacuo, dissolved in DMSO at $20^{\circ} \mathrm{C}$, reprecipitated, rewashed with methanol and dried in

Table I. Acetylation condition, $\langle\mathrm{F}\rangle$, and $\left\langle\mathrm{f}_{k}\right\rangle$ of CAs (one-step)

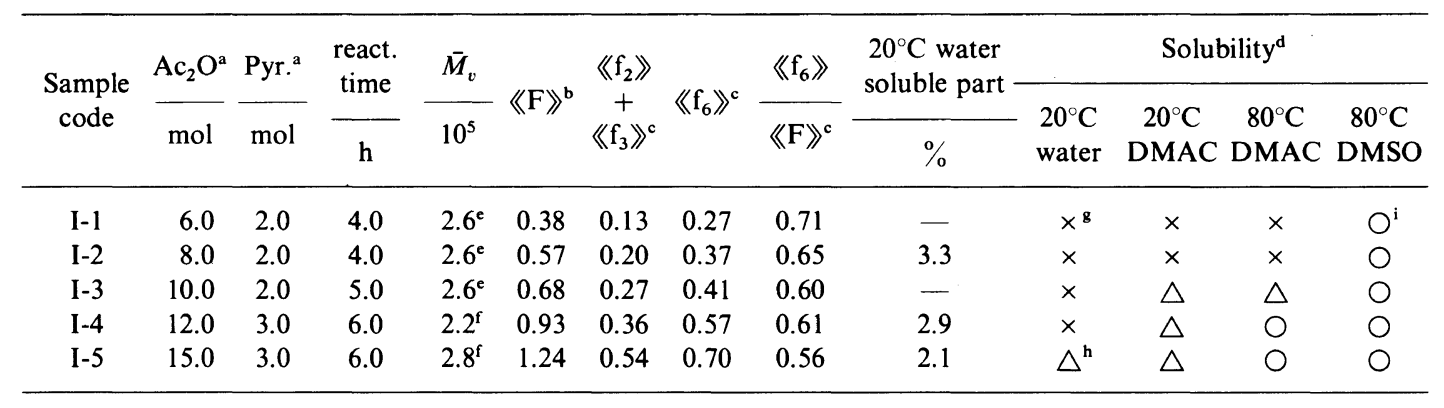

${ }^{a} \mathrm{~mol} \mathrm{~mol}^{-1}$ glucose unit. ${ }^{\mathrm{b}}$ From titration. ${ }^{\mathrm{c}}$ From NMR. d Judged visually. e From [ $\left.\eta\right]$ in DMSO.

${ }^{f}$ From $[\eta]$ in DMAC. ${ }^{g}$ Insoluble. ${ }^{\mathrm{h}}$ Highly swelling. ${ }^{\mathrm{i}}$ Soluble. 
Solubility of Cellulose Acetates

Table II. $\bar{M}_{w},\left\langle\mathrm{~F} 》\right.$, and $\left\langle\mathrm{f}_{k}\right\rangle$ of CAs (two-step)

\begin{tabular}{|c|c|c|c|c|c|c|c|c|c|}
\hline \multirow{2}{*}{$\begin{array}{l}\text { Sample } \\
\text { code }\end{array}$} & \multirow{2}{*}{$\frac{\bar{M}_{w}\left(\bar{M}_{v}\right)}{10^{5}}$} & \multirow{2}{*}{$\langle\langle F\rangle\rangle^{a}$} & \multirow{2}{*}{$\left.\left\langle\mathrm{f}_{2}\right\rangle\right\rangle+\left\langle\left\langle\mathrm{f}_{3}\right\rangle^{b}\right.$} & \multirow{2}{*}{$\left\langle\mathrm{f}_{6}\right\rangle^{b}$} & \multirow{2}{*}{$\left\langle\left\langle\mathrm{f}_{6}\right\rangle /\langle\langle\mathrm{F}\rangle\rangle^{b}\right.$} & \multirow{2}{*}{$\begin{array}{c}20^{\circ} \mathrm{C} \text { water } \\
\text { soluble part }\end{array}$} & \multicolumn{3}{|c|}{ Solubility ${ }^{\mathfrak{c}}$} \\
\hline & & & & & & & $\begin{array}{l}20^{\circ} \mathrm{C} \\
\text { water }\end{array}$ & $\begin{array}{c}20^{\circ} \mathrm{C} \\
\text { DMAC }\end{array}$ & $\begin{array}{c}80^{\circ} \mathrm{C} \\
\mathrm{DMSO}\end{array}$ \\
\hline СТA- 0 & 2.32 & 2.92 & 1.89 & 1.03 & 0.35 & 0 & $x^{e}$ & $O^{f}$ & 0 \\
\hline II-1 & 1.20 & 2.46 & 1.59 & 0.86 & 0.35 & 0 & $x$ & 0 & 0 \\
\hline II-2 & $0.46^{d}$ & 1.75 & 1.14 & 0.61 & 0.35 & 0 & $x$ & 0 & 0 \\
\hline II-3 & $0.41^{\mathrm{d}}$ & 0.80 & 0.53 & 0.27 & 0.34 & 100 & 0 & 0 & 0 \\
\hline II-4 & 0.80 & 0.68 & 0.45 & 0.23 & 0.34 & 100 & 0 & 0 & 0 \\
\hline
\end{tabular}

${ }^{\text {a }}$ From titration. ${ }^{\mathrm{b}}$ From NMR. ${ }^{\mathrm{c}}$ Judged visually. ${ }^{\mathrm{d}} \bar{M}_{\boldsymbol{r}}$ from $[\eta]$ in DMAC. ${ }^{\mathrm{e}}$ Insoluble. ${ }^{\mathrm{f}}$ Soluble.

vacuo again. The five samples prepared in this way were subjected to further NMR measurements. In the fifth column of the table, the viscosity-average molecular weight $\bar{M}_{v}$ of the samples, estimated from the hypothetical MHS equation of CA/DMAC or CA/DMSO system at $25^{\circ} \mathrm{C}$, is compiled. The $k$ and $a$ values in the MHS equations for estimating $\bar{M}_{v}$ for CA having given $\langle\mathrm{F}\rangle$ were derived from correlationships between $\langle\mathrm{F}\rangle$ and $k, a$ values in the MHS equations for various CA established by Kamide et al. ${ }^{7.10-12}\langle\mathrm{~F}\rangle$, determined by the neutralization titration method, of these CA samples is also given in the sixth column of the table.

Two-Step Method. An unfractionated cellulose triacetate sample with $\langle\mathrm{F}\rangle$ of 2.92 was deacetylated in acidic media in the same manner, as described in the previous papers ${ }^{7.10-12}$ to obtain four CA samples with different $\langle\bar{F}\rangle$. Table II summarizes the data of the weightaverage molecular weight $\bar{M}_{n^{\prime}}$ by the light scattering method and $\langle\mathrm{F}\rangle$ by the titration method of four CA samples prepared by the two-step method. In the table, the data on the original CTA sample are also included.

\section{${ }^{13}$ C Nuclear Magnetic Resonance Spectra}

Proton-noise-decoupled ${ }^{13} \mathrm{C}$ NMR spectra were recorded for all ten $\mathrm{CA}$ samples in DMSO- $d_{6}$ at the polymer concentration of $7 \mathrm{wt} \%$ at $50.15 \mathrm{MHz}$ on a JEOL FX-200 pulseFourier-transform NMR spectrometer at $100^{\circ} \mathrm{C}$. Here, the detailed operating conditions are the same as used before. ${ }^{6}$ Slight decrease in solution viscosity was observed during NMR measurements of $14 \mathrm{~h}$, implying that CA degradated to some extent in DMSO at $100^{\circ} \mathrm{C}$.

\section{Solubility Determination}

To $0.25 \mathrm{~g}$ of CA sample $5 \mathrm{ml}$ of distilled water was added at room temperature. The mixture was stirred using a home mixer, subjected to centrifuge and the insoluble part was dried in vacuo and weighed. The weight percentage of the water-soluble part of CA is indicated in the tenth column of Table I and the seventh column of Table II. The watersolubility was also judged in a qualitative manner visually. As was also the solubility of CA against DMAC and DMSO. The results are compiled in the last columns of Tables I and II.

\section{RESULTS AND DISCUSSION}

Figure 1 shows the ${ }^{13} \mathrm{C}\left\{{ }^{1} \mathrm{H}\right\}$ NMR spectra in $O$-acetyl carbonyl carbon region of CTA $\langle\mathrm{F}\rangle=2.92$ ) and CA (one-step method, sample code I-1-I-5). For CTA, three large and sharp peaks, attributable to the substituent groups at $\mathrm{C}_{6}, \mathrm{C}_{3}$, and $\mathrm{C}_{2}$ positions in trisubstituted (i.e., fully acetylated) glucopyranose unit, were observed, as noted before, at $169.8,169.0$, and $168.6 \mathrm{ppm}$, respectively. For CA (one-step method, $\langle F\rangle=0.38$ ), three 


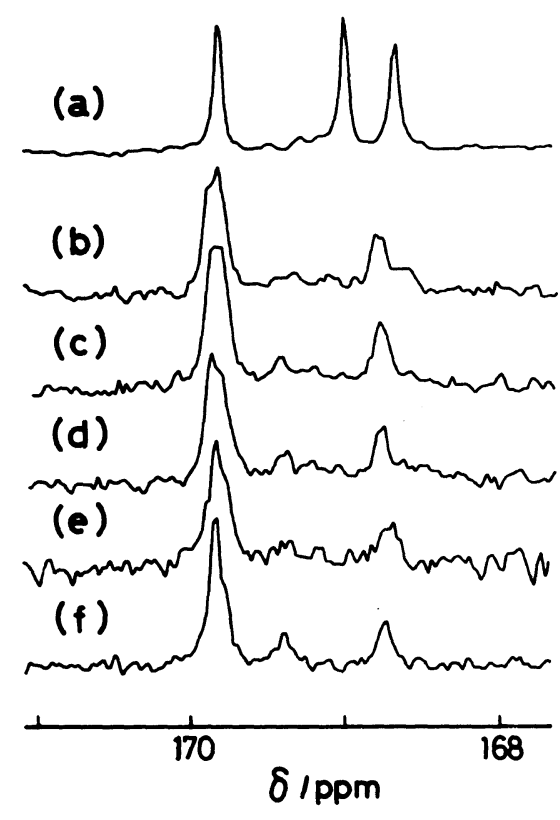

Figure 1. ${ }^{13} \mathrm{C}\left\{{ }^{1} \mathrm{H}\right\}$ NMR spectra in $O$-acetyl carbonyl carbon region of CTA and CA (one-step method) in DMSO- $d_{6}$. (a), CTA-0 $(《 \mathrm{~F}\rangle=2.92$; (b), I-5 (1.24); (c), I4 (0.93); (d), I-3 (0.68); (e), I-2 (0.57); (f), I-1 (0.38).

peaks were observed at $169.9,169.4$, and 168.8 ppm and they can be assigned for the $O$-acetyl carbonyl carbon of $\mathrm{C}_{6}$-mono-, $\mathrm{C}_{3}$-mono-, and $\mathrm{C}_{2}$-mono-substituted glucopyranose units, respectively. It is obvious from Figure 1 that the difference in chemical shift of $O$-acetyl carbonyl carbon at $C_{6}$ or $C_{2}$ position is only of the order of $0.1-0.2 \mathrm{ppm}$ between tri-substituted and mono-substituted glucopyranose units (that is, the $O$-acetyl carbonyl carbon of trisubstituted glucopyranose units is located at higher magnetic field than that of monosubstituted ones). But, the situation is somewhat different for the carbonyl carbon at $\mathrm{C}_{3}$ position. There is a large $(0.4 \mathrm{ppm})$ change between tri- and mono-substituted glucopyranose units.

CA samples with $\langle F\rangle=0.57-1.24$ can have di-substituted glucopyranose units. $O$ acetyl carbonyl carbon peaks on $\mathrm{C}_{6}$ position for $\mathrm{C}_{6}, \mathrm{C}_{2}$-di- and $\mathrm{C}_{6}, \mathrm{C}_{3}$-di-substituted ones may appear around $169.8 \mathrm{ppm}\left(\mathrm{C}_{6}\right.$ in tri-

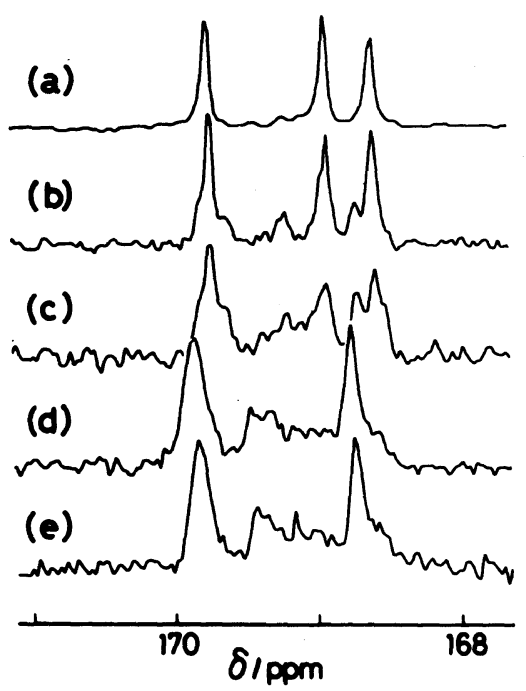

Figure 2. ${ }^{13} \mathrm{C}\left\{{ }^{1} \mathrm{H}\right\}$ NMR spectra in $O$-acetyl carbonyl carbon region of CTA and CA (two-step method) in DMSO- $d_{6}$. (a), CTA-0 (《F $\left.\rangle=2.92\right)$; (b), II-1 (2.46); (c), II-4 (1.75); (d), II-3 (0.80); (e), II-4 (0.68).

substituted one) because additional substitution to $\mathrm{C}_{2}$ or $\mathrm{C}_{3}$ may not influence greatly the $O$-acetyl carbonyl carbon at $\mathrm{C}_{6}$ magnetically. Complicated peaks between 169.0 and 169.7 ppm might originate from $O$-acetyl carbonyl carbons at $\mathrm{C}_{3}$ and possibly at $\mathrm{C}_{2}$ for $\mathrm{C}_{3}, \mathrm{C}_{6}$-di- and $\mathrm{C}_{2}, \mathrm{C}_{3}$-di-substituted ones.

Figure 2 shows the ${ }^{13} \mathrm{C}\left\{{ }^{1} \mathrm{H}\right\}$ NMR spectra of $\mathrm{O}$-acetyl carbonyl carbon region of CTA $(\langle F\rangle=2.92)$ and CA (two-step method, sample code II-1-II-4. The spectra conspicuously changed with decreasing $\langle F\rangle$. When $\langle F\rangle$ decreased from 2.92 to 2.46 , peaks appeared at 169.7, 169.2, and $168.8 \mathrm{ppm}$. These envelopes may originate from $O$-acetyl carbonyl carbons on $\mathrm{C}_{6}, \mathrm{C}_{3}$, and $\mathrm{C}_{2}$ for disubstituted glucopyranose units, respectively. For $C A$ with $\langle F\rangle=0.68$, which may be mainly constituted of mono-substituted ones, $O$ acetyl carbonyl carbon peaks of $\mathrm{C}_{6}$-mono-, $\mathrm{C}_{3}$ mono-, $\mathrm{C}_{2}$-mono-substituted at $169.9,169.4$, and $168.8 \mathrm{ppm}$ were again clearly observed. It should be noted again here that the spectra patterns between 169.0 and 169.7 ppm are very complicated especially for CA with $\langle\mathrm{F}\rangle=0.68$ 
and 0.80 . Assuming that the peak positions of the carbonyl carbon peaks due to disubstituted glucopyranose units are not far from the corresponding peaks of mono- and tri-substituted glucopyranose units, we can divide roughly the whole spectra in a carbonyl carbon region into three regions: the region between $170-169.6 \mathrm{ppm}$ for $\mathrm{C}_{6}, 169.6-168.9$ ppm for $\mathrm{C}_{3}$, and $168.9-168.3 \mathrm{ppm}$ for $\mathrm{C}_{2}$. It should be remembered that this technique might give a convenient basis for estimating 《 $\left.\mathrm{f}_{k}\right\rangle$ from ${ }^{13} \mathrm{C}$ NMR spectra, but it is not so rigorous. For example, two peaks groups assigned for $\mathrm{C}_{3}$ and $\mathrm{C}_{2}$ positions have an innegligible possibility of mutual overlapping, which can be realized, for example, by noting that at least more than 6 peaks are observed experimentally in the above defined carbonyl carbon region at the so-called $\mathrm{C}_{3}$ position, although the maximum number of corresponding peaks, theoretically expected, is only four (one for mono-, for tri-, and two for disubstituted glucopyranose units). Here, the theoretical number of peaks is taken by assuming that neighbouring glucopyranose unit does not affect $O$-acetyl carbonyl carbon on $\mathrm{C}_{3}$ in question. In contrast to this, the carbonyl carbon peak group at $\mathrm{C}_{6}$ position is absolutely separated from those at other positions. Then, $\left\langle\mathrm{f}_{6}\right\rangle$ and $\left(\left\langle\mathrm{f}_{2}\right\rangle+\left\langle\left\langle\mathrm{f}_{3}\right\rangle\right)\left(=\langle\mathrm{F}\rangle-\left\langle\left\langle\mathrm{f}_{6}\right\rangle\right)\right.\right.$ can be accurately estimated, regardless of its $\langle\mathrm{F}\rangle$, from ${ }^{13} \mathrm{C}$ NMR carbonyl carbon region spectra of $C A$ with help of $\langle F\rangle$ (by the titration method).

Comparison of Figures 1 and 2 indicates that the probability of substituting the hydroxyl group at $\mathrm{C}_{6}$ position with acetyl group is higher in one-step method for CA with same $\langle F\rangle$. Below $\langle F\rangle=1.00$, the probability of substitution at $\mathrm{C}_{3}$ or fraction of $\mathrm{C}_{3}$-monosubstituted one for one-step CA is far lower than that of two-step CA.

In Tables I and II, $\left\langle\mathrm{f}_{6}\right\rangle$ and $\left(\left\langle\mathrm{f}_{2}\right\rangle+\left\langle\left\langle\mathrm{f}_{3}\right\rangle\right)\right.$ data for all CA samples thus estimated are collected.

Figure 3 shows the plot of $\left\langle\mathrm{f}_{6}\right\rangle$ against $\langle F\rangle$

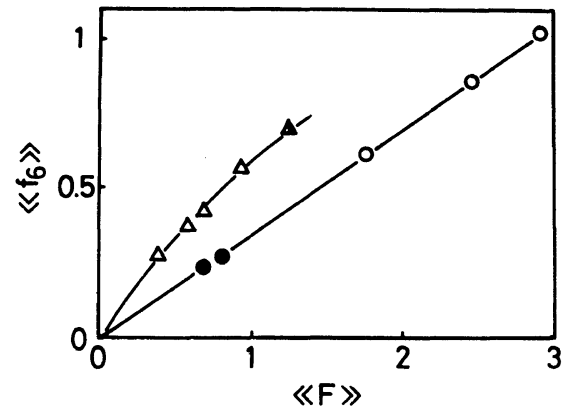

Figure 3. Plot of $\left\langle\mathrm{f}_{6}\right\rangle$ of $C A$ against $\langle F\rangle$. $(\triangle, \Delta)$, one-step method; $(O, \bigcirc)$, two-step method; unfilled marks, water-insoluble; half-filled marks, highly swelling in water; filled marks, water-soluble.

for two series of CA samples (one-step and two-step methods). In the figure, the unfilled mark denotes water-insoluble and the halffilled mark, partially soluble and highlyswelling in water and the filled mark, completely water-soluble. From Tables I and II together with Figure 3, the following relations hold: $\left\langle\mathrm{f}_{6}\right\rangle \mid\langle\mathrm{F}\rangle=0.65 \pm 0.06$ for $\mathrm{CA}$ (one-step method) and $\left\langle\mathrm{f}_{6}\right\rangle \simeq\left(\left\langle\mathrm{f}_{2}\right\rangle+\left\langle\mathrm{f}_{3}\right\rangle\right) / 2$ for $\mathrm{CA}$ (two-step method).

In the one-step method, the hydroxyl group at $\mathrm{C}_{6}$ position is $3-4$ times reactive against acetic anhydride than that at $\mathrm{C}_{2}$ or $\mathrm{C}_{3}$ position. Whereas, in the two-step method, the acetyl groups at three different carbon positions are almost equally hydrolyzed when dissolved in acidic media. In other words, the reactivity of acetylation depends strongly on the carbon positions, to which the hydroxyl group is located, but that of deacetylation does not significantly depend on whether the acetyl group is derived from the primary hydroxyl or secondary group.

All CA samples in this paper are soluble in DMSO at $80^{\circ} \mathrm{C}$ and CA samples prepared in two-step method are also soluble in DMAC at $20^{\circ} \mathrm{C}$. For many years, we have regarded DMAC as a common solvent for CA having various $\langle F\rangle$ and in fact the measurements of the solution properties of CA have been carried out in the DMAC system. But, CA sam- 
ples prepared in the one-step method were found not to dissolve in DMAC at $20^{\circ} \mathrm{C}$. This is the first demonstration of the solubility study on CA. It is also evident from the tables that the solubility of CA samples against water and DMAC is different from sample to sample. Our previous study $^{7}$ revealed that $\bar{M}_{w}$ dependence of solubility of CA prepared in the two-step method was not found up to $\bar{M}_{w}=$ $1.45 \times 10^{5}$, showing that $\bar{M}_{w}$ is not essential for the solubility of CA.

A comparison of sample codes I-1 and II-3, both having the same $\left\langle\mathrm{f}_{6}\right\rangle(=0.27)$ and different $\langle\vec{F}\rangle$, shows that even if $\left\langle\left\langle\mathrm{f}_{6}\right\rangle\right.$ is the same the solubility against water and DMAC is better for the polymer having larger $\left(\left\langle\mathrm{f}_{2}\right\rangle\right\rangle+\left\langle\left\langle\mathrm{f}_{3}\right\rangle\right)$. A comparison of sample codes I-3 and II-4 leads us to the conclusion that, CA with larger $\left.\left(\left\langle\mathrm{f}_{2}\right\rangle\right\rangle+\left\langle\mathrm{f}_{3}\right\rangle\right)$ exhibits a better solubility if compared at the same $\langle F\rangle$. Therefore, it seems doubtlessly reasonable to consider that the solubility of CA is predominantly governed by $\left(\left\langle\mathrm{f}_{2}\right\rangle\right\rangle+\left\langle\left\langle\mathrm{f}_{3}\right\rangle\right)$. However, CA samples (I-5, II-3) having almost same $\left(\left\langle\mathrm{f}_{2}\right\rangle\right)+\left\langle\left\langle\mathrm{f}_{3}\right\rangle\right)(\simeq 0.54)$ have different solubility against water and DMAC. As mentioned before, CA sample codes II-3 which are completely water-soluble have higher probability of substitution at $\mathrm{C}_{3}$ or higher fraction of $\mathrm{C}_{3}$-mono-substituted glucopyranose units than CA sample codes I-5. Therefore, the minimum necessary conditions in which the CA sample dissolves in water and polar solvent such as DMAC seems to be that the degree of substitution at $\mathrm{C}_{3}$ should be higher than a given threshold value. The threshold value cannot be exactly estimated but from Figures 1 and 2 it may be around 0.2 for water. That is, substitution at $\mathrm{C}_{3}$ is one/five glucopyranose units. The introduction of an hydrophobic group at $\mathrm{C}_{3}$ may break down the intramolecular hydrogen bond between $\mathrm{O}_{3} \cdots \mathrm{O}_{5}$. For two series of the CA sample, the solubility power of DMAC is somewhat larger than water. This may be due to the difference in solvation power to $O$-acetyl and hydroxyl groups of the solvents. These experimental results agree well with those by Miyamoto et $a l^{8}$ who demonstrated that CA having higher $\left\langle\mathrm{f}_{6}\right\rangle$ than $\left\langle\mathrm{f}_{2}\right\rangle$ and $\left\langle\mathrm{f}_{3}\right\rangle$, synthesized by one-step method (i.e., A series samples in their paper), does not dissolve in water at room temperature and they proposed that $\left\langle\mathrm{f}_{2}\right\rangle$ and $\left\langle\mathrm{f}_{3}\right\rangle$ play key roles in the watersolubility of CA. But, they did not give any explanation for their results. Note that $\left.\left(\left\langle\mathrm{f}_{2}\right\rangle\right)+\left\langle\mathrm{f}_{3}\right\rangle\right)$ or $\left.\left\langle\mathrm{f}_{3}\right\rangle\right\rangle$ or fraction of $\mathrm{C}_{3}$-monosubstituted glucopyranose units is, of course, not a single parameter controlling water solubility.

Concerning the reactivity of cellulose towards acetic anhydride in $\mathrm{DMAC} / \mathrm{LiCl}$ medium, Miyamoto et al. ${ }^{8}$ considered that the strength of hydrogen bonding by $\mathrm{C}_{2}$ and $\mathrm{C}_{3}$ hydroxyl groups plays an important role, as compared with that of $\mathrm{C}_{6}$ hydroxyl group. They base their discussion on Gagnaire et al.'s claim ${ }^{13}$ that the intramolecular hydrogen bonding between the hydroxyl group at $\mathrm{C}_{3}$ position and a heterocyclic oxygen atom in neighbouring glucopyranose unit of cellulose may exist even in the solution. Note that Gagnaire et al.'s assertion was formed only indirectly by observing the constancy of ${ }^{1} \mathrm{H}$ NMR spectra peak position of hydroxyl proton at $\mathrm{C}_{3}$ position $(4.6 \mathrm{ppm})$ of cellobiose (not cellulose!) dissolved in dimethylacetamide (DMAC)/LiCl system. It is well-recognized that ${ }^{13} \mathrm{C}$ NMR information on polysaccharides depends significantly on the degree of polymerization (DP) in an extremely low DP region. Kamide and Saito ${ }^{14}$ showed experimentally, that the unperturbed chain dimension of cellulose derivatives, including CA, increases with the polarity of the solvent (dielectric constant $\varepsilon$ ) and both the substituent groups and remaining hydroxyl group of cellulose derivatives solvate with the solvent and the degree of solvation is closely correlated with $\varepsilon$. These facts explicitly demonstrate the impossibility of the existence of the intramolecular hydrogen bonding of $\mathrm{CA}$ in the 
solvents. Accordingly, the selective acetylation at $\mathrm{C}_{6}$ position for the cellulose in $\mathrm{DMAC} / \mathrm{LiCl}$ system does not indicate difference in strength of intramolecular hydrogen bondings between the hydroxyl groups at $\mathrm{C}_{2}, \mathrm{C}_{3}$, and $\mathrm{C}_{6}$ positions and a heterocyclic oxygen atom. The experimental data, summarized in Table II, suggest that a primary hydroxyl group at $\mathrm{C}_{6}$ position is highly reactive to an acetylation reagent than secondary hydroxyl groups at $\mathrm{C}_{2}$ and $\mathrm{C}_{3}$ positions as in the case of low molecular weight organic compounds. ${ }^{15}$

Kamide et al. ${ }^{3}$ demonstrated that, as long as $\mathrm{NaCMC}$ does not dissolve in the liquids, the absorbency can be accurately determined by $\left\langle\mathrm{f}_{6}\right\rangle$ alone. They explained this fact as follows: the introduction of a bulky substituent (carboxymethyl group) at the $\mathrm{C}_{6}$ position destroys the intermolecular hydrogen bonds between the hydroxyl group at $\mathrm{C}_{6}$ position and a heterocyclic oxygen atom in a neighbouring glucopyranose unit and also widens the distance between molecular chains, resulting in destruction of the intermolecular hydrogen bonds, creating a wide space to readily receive the absorbed liquids. It should be noted that the results by Kamide et $a l .^{3}$ for NaCMC do not mean that the simple introduction of the bulky substituent group into $\mathrm{C}_{6}$ position is an important step to make cellulose soluble in water or aqueous solutions. Carboxymethyl group has the same molecular volume as an acetyl group and then, the introduction of these groups into $\mathrm{C}_{6}$ positions is expected to bring about the destruction of intermolecular hydrogen bonding to the same degree. If the destruction of hydrogen bonding associated with $\mathrm{C}_{6}$ position is a single universal factor controling the water-solubility, CA with high $\left\langle\mathrm{f}_{6}\right\rangle$ is should be water soluble. Unfortunately, this is not true. It is interesting to note that for $\mathrm{NaCMC}$, the carboxymethyl group is more hydrophilic than the hydroxyl group and in CA the acetyl group is less hydrophilic than the hydroxyl group. Then, the affinity of the substituent group against water seems another important factor.

Of course, an attempt to directly correlate the chemical structure, as characterized in terms of $\left\langle\mathrm{f}_{2}\right\rangle$, $\left\langle\left\langle\mathrm{f}_{3}\right\rangle\right.$, and $\left\langle\left\langle\mathrm{f}_{6}\right\rangle\right.$, of cellulose derivatives with their solubility is too simple and rough. The solubility should be mainly determined by the supermolecular structure of the solid (i.e., the crystalline and amorphous phases, intra- and intermolecular hydrogen bonds) and the latter is vitally controlled by the chemical structure, provided that the heterogeneity of the chemical structure in glucopyranose unit can be ignored and the solid is formed by a similar procedure. Recently, Kamide et al. ${ }^{16}$ pointed out that the solubility of cellulose in a $10 \mathrm{wt} \%$ aqueous $\mathrm{NaOH}$ at $4{ }^{\circ} \mathrm{C}$ could generally be correlated to the relative amount of the high magnetic field envelope of $\mathrm{C}_{4}$ carbon NMR peak (i.e., that of the region where intramolecular hydrogen bonds are at least partly broken). At the present time, the supermolecular structures of CA samples are not fully understood and remain open for further study.

\section{REFERENCES}

1. K. Kamide, K. Okajima, T. Matsui, M. Ohnishi, and H. Kobayashi, Polym. J., 15, 309 (1983).

2. K. Kamide, K. Okajima, T. Matsui, and $\mathrm{H}$. Kobayashi, Polym. J., 16, 259 (1984).

3. K. Kamide, K. Okajima, K. Kowsaka, T. Matsui, S. Nomura, and K. Hikichi, Polym. J., 17, 909 (1985).

4. K. Kamide and K. Okajima, Polym. J., 13, 127 (1981).

5. T. Miyamoto, Y. Sato, T. Shibata, H. Inagaki, and M. Tanahashi, J. Polym. Sci., Polym. Chem. Ed., 22, 2363 (1984)

6. K. Kowsaka, K. Okajima, and K. Kamide, Polym. J., 18, 843 (1986).

7. K. Kamide, M. Saito, and T. Abe, Polym. J., 13, 421 (1981).

8. T. Miyamoto, Y. Sato, T. Shibata, M. Tanahashi, and H. Inagaki, J. Polym. Sci., Polym. Chem. Ed., 23, 1373 (1985).

9. W. Brown and R. Wikström, Eur. Polym. J., 1, 1 (1966).

10. K. Kamide, T. Terakawa, and Y. Miyazaki, Polym. J., 11, 285 (1979).

11. K. Kamide, T. Miyazaki, and T. Abe, Polym. J., 11, 523 (1979). 
12. M. Saito, Polym. J., 15, 249 (1983).

13. D. Gagnaire, J. Saint-Germain, and M. Vincendon, J. Appl. Polym. Sci., Polym. Symp., 37, 261 (1983).

14. K. Kamide and M. Saito, Eur. Polym. J., 20, 903 (1984).
15. For example, J. Roberts, R. Stewart, and M. Caserio, "Organic Chemistry," W. A. Benjamin, Inc., Menro Park, Cal., 1973.

16. K. Kamide, K. Okajima, T. Matsui, and K. Kowsaka, Polym. J., 12, 857 (1984). 